\title{
Les nouveaux éditeurs russes
}

Anne-Marie Thiesse, Madame Natalia Chmatko

\section{Citer ce document / Cite this document :}

Thiesse Anne-Marie, Chmatko Natalia. Les nouveaux éditeurs russes. In: Actes de la recherche en sciences sociales. Vol. 126-127, mars 1999. Édition, Éditeurs (1) pp. 75-89;

doi : https://doi.org/10.3406/arss.1999.3283

https://www.persee.fr/doc/arss_0335-5322_1999_num_126_1_3283

Fichier pdf généré le 22/03/2019 


\section{Résumé}

Les nouveaux éditeurs russes.

Depuis la disparition du monopole étatique et la levée de la censure, des changements radicaux ont affecté l'édition russe. De nombreuses maisons d'édition privées ont été créées. Les nouveaux éditeurs russes sont en majorité des individus dotés d'un fort capital culturel qu'ils tentent de réinvestir dans la configuration sociale et économique chaotique qui caractérise la Russie actuelle. La production de livres, désormais dépendante des possibilités de vente à très court terme, a été profondément modifiée, quantitativement (baisse globale, mais inégale selon les genres, des tirages) et qualitativement. De nouveaux genres sont apparus (ouvrages religieux, par exemple) ou se sont considérablement développés (roman populaire) alors que les publications scientifiques ou à forte valeur culturelle sont en déclin. Si la politique éditoriale des différentes maisons a été déterminée dans une première phase par la nécessité de constituer par tous les moyens possibles un capital économique, une certaine polarisation du secteur éditorial se dessine, qui oppose en ses extrémités l'édition dite commerciale (romans à grosse vente) et la production pour producteurs. L'absence de véritables structures de distribution, l'appauvrissement de la majeure partie de la population, les problèmes de financement des bibliothèques publiques fragilisent l'édition. L'espace de production et de diffusion des livres se restreint de plus en plus aux deux principales villes russes.

\section{Resumen}

Los nuevos editores rusos

Desde que desapareció el monopolio estatal y se dejó de ejercer la censura, la edición rusa ha sufrido cambios radicales. Se han creado muchas editoriales privadas. En su mayoría, los nuevos editores rusos poseen una gran cultura personal, capital que intentan invertir en la caótica configuración social y económica que caracteriza a la Rusia actual. La producción de libros, que hoy día depende de las posibilidades de venta a muy corto plazo, se ha modificado profundamente, tanto cualitativa como cuantitativamente (disminución global de las tiradas, aunque con diferencias según los géneros). Han aparecido nuevos géneros (por ejemplo libros religiosos) y otros se desarrollan considerablemente (novela popular) ; al mismo tiempo, las publicaciones científicas y aquéllas de alto valor cultural están en decadencia. En una primera fase, la política de las diferentes editoriales estuvo determinada por la necesidad de constituir, por todos los medios, un capital económico. Actualmente se nota una cierta polarización del sector editorial, que en sus extremos opone la llamada edición comercial (novelas de mucha venta) a la producción para productores. La ausencia de verdaderas estructuras de distribución, el empobrecimiento de la mayor parte de la población y los problemas de financiamiento de las bibliotecas públicas debilitan la edición. La producción y la difusión de libros se reduce cada vez más al área de las dos principales ciudades rusas.

\section{Zusammenfassung}

Die neuen russischen Verleger

Seit Abschaffung des staatlichen Monopols und der Zensur hat das russische Verlagswesen einschneidende Veränderungen erfahren. Zahlreiche private Verlage sind entstanden. Die neuen russischen Verleger verfügen in der Regel über bedeutendes kulturelles Kapital, das sie in den chaotischen sozialen und ökonomischen Bedingungen des heutigen Rulslands nutzbringend anzuwenden versuchen. Nunmehr auf die Realisierung kurzfristiger Gewinne angewiesen, hat die Bücherproduktion sich eben sowohl der Quantität (insgesamtes, dabei nach Gattungen unterschiedlich starkes Schrumpfen der Umsatze), wie der Qualität nach grundlegend modifiziert. Neue Gattungen (wie etwa religiöses Schrifttum) sind aufgetaucht, andere (z. B. Trivialliteratur) haben erheblich zugenommen, demgegenüber sind wissenschaftlich und kulturell hochwertige Veröffentlichungen zurückgegangen. Waren in einer ersten Phase die Strategien der verschiedenen Verlagshäuser davon bestimmt gewesen, auf jede nur erdenkliche Weise ökonomisches Kapital anzuhaufen, so ist jetzt eine gewisse Polarisierung des Büchermarktes festzustellen, bei der am einen Extrem sogenannte kommerzielle Editionen (d.h. Romane hoher Auflagen), am anderen Produktionen fur Produkteure zu finden sind. Das Fehlen echter Vertriebsstrukturen, die finanziellen Schwierigkeiten des größten Teils der Bevölkerung und der Geldmangel öffentlicher Bibliotheken wirken sich nachteilig auf das Verlagswesen aus. Der Herstellungs- und Vertriebsraum schränkt sich zunehmend auf die beiden 
bedeutendsten russischen Stadte ein.

\section{Abstract}

The new Russian publishers

Since the disappearance of the State monopoly and censorship, Russian publishing has changed radically. Many private publishing houses have sprung up. On the whole, the new Russian publishers are people with an important cultural capital which they are trying to reinvest in the chaotic social and economic configuration characteristic of present-day Russia. Book production, which now depends on potential sales in the very short term, has been thoroughly modified, both quantitatively (overall lower runs, but this depends on the field) and qualitatively. New fields have appeared (religions books, for example) or have developed considerably (popular novels), while scientific works or titles with a strongly cultural value are in decline. If the editorial policy of the different houses was initially determined by the need to build up an economic capital by any means available, a certain polarization can now be seen in the editorial sector which opposes, at one end, the so-called commercial publication (best-sellers) to producers production, at the other end. The absence of any true distribution networks, the impoverishment of the bulk of the population, the lack of funding for public libraries combine to sap the strength of the publishing world. Book production and distribution are increasingly restricted to the two largest cities.

In an attempt to understand the transformations that have occurred in the field of publishing in Brazil, the author traces the history of the Jose Olympio publishing hou.se. Founded in the 1930s, it played a central role in building the national cultural by publishing, in addition to the books for a large audience, young littleknown authors, in the framework of a family-like company spirit. Confron-ted with the internationalization of the book market and its commercialization, the only way to stem the compa-ny's decline was to change the organization of the work and to introduce an entrepreneurial, bureaucratie and rational logic. The publisher thus found himself forced to become a true " professional of the book », quite far from the literary images often associated with the job. 


\section{Anne-Marie Thiesse et Natalia Chmatko}

\section{LES NOUVEAUX ÉDITEURS RUSSES}

$\mathrm{L}$ a production culturelle était en URSS un enjeu majeur pour le pouvoir. Parce qu'elle était invesétait à la fois hautement valorisée et étroitement contrôlée. Chargée officiellement d'une fonction politique et idéologique, elle était aussi le seul lieu d'une expression contestataire que l'hégémonie du parti unique ne permettait pas d'exposer autrement. Avec l'effondrement brutal du communisme et la levée de la censure, l'activité culturelle et intellectuelle a été affranchie de ce rapport étroit au politique. Mais se pose du même coup la redéfinition de sa légitimité et de sa fonction, alors que les changements politiques et économiques modifient radicalement ses conditions d'exercice et font apparaître de nouveaux critères d'évaluation.

Tout le système des positions et des dispositions associées qui caractérisait la société soviétique a été frappé par le changement. Le capital culturel anciennement valorisé se trouve soudainement démonétisé, sauf à être renégocié dans le nouvel espace où la détention de capital économique joue désormais un rôle déterminant. L'évolution de l'activité éditoriale russe ${ }^{1}$, depuis 1990, résulte à la fois du désengagement du pouvoir en matière de production culturelle et de stratégies de reconversion menées par des agents qui, en entrant temporairement ou durablement dans ce secteur, tentent d'investir au meilleur taux les dispositions et capitaux immatériels dont ils sont pourvus. Cependant, ces investissements ne peuvent s'inscrire que dans des stratégies à court terme. Le fonctionnement et les dysfonctionnements de l'édition russe actuelle tiennent à une situation d'entre-deux, d'économie non régulée née sur les décombres du monopole étatique. La chute des tirages a été drastique, tandis que se multipliaient les créations de maisons d'édition. Si une tendance à la concentration est apparue, elle a pris plutôt forme géo- graphique : l'espace de production et de diffusion de livres s'est restreint progressivement aux deux plus grandes villes. L'édition russe, à la fin des années 1990, se présente sous forme d'une multitude de petites entreprises parmi lesquelles règne une intense concurrence. Alors que dans une phase initiale, durant les premières années de la décennie, l'édition était aussi vivante qu'anarchique, n'importe qui publiant n'importe quoi, une polarisation de l'ensemble s'est esquissée, qui oppose en ses extrémités la production de grande diffusion et la production restreinte, quelques maisons essayant d'occuper l'espace médian et de se présenter comme généralistes.

\section{LE LIVRE SOVIÉTIQUE: PÉNURIE DANS L'ABONDANCE}

À l'époque soviétique, la Russie comptait 300 maisons d'édition qui appartenaient à l'État ou à des organisations centrales (Union des syndicats, Union des jeunesses communistes, Académie des sciences). Ces maisons étaient assez précisément spécialisées dans un genre de production (belles-lettres, sciences, manuels scolaires, etc.) et n'entraient pas en concurrence les unes avec les autres. Elles n'étaient pas véritablement tenues de prendre en considération la rentabilité de leur production puisque leur budget de fonctionnement était assuré par l'État ou l'organisme propriétaire. En principe, les ventes devaient couvrir les dépenses, mais des subventions compensaient les pertes éventuelles. Une maison d'édition était souvent associée à

1 - La recherche de terrain a été conduite par Natalia Chmatko entre 1995 et 1998 . L'Observatoire France-Loisirs de la lecture a apporté un financement à ce travail entre juin 1997 et décembre 1998. 
une ou plusieurs imprimeries, ainsi qu'à un ensemble de librairies. Les Éditions Naouka, par exemple, qui appartenaient à l'Académie des sciences, avaient trois imprimeries et des librairies, dont six pour la seule ville de Moscou. Comme.c'était souvent le cas pour les entreprises soviétiques, beaucoup de maisons d'édition disposaient non seulement de locaux pour leurs activités, mais aussi de logements pour le personnel et de centres de vacances.

L'ensemble des entreprises du secteur du livre, imprimeries, réseaux de distribution et librairies, appartenaient également à l'État ou aux organismes centraux et relevaient donc du même cadre de non-concurrence, de non-obligation de rentabilité et d'observation des directives de la planification. Plusieurs organismes centralisés étaient chargés de la distribution. Le plus important était le réseau Soyouzkniga, disposant d'un dépôt central et de dépôts régionaux et locaux. Il effectuait des envois de livres en fonction des commandes passées par les distributeurs locaux (dépôts secondaires, librairies, universités, écoles). Du fait de cette organisation, on pouvait d'ailleurs trouver en province des livres rares ou épuisés dans les grandes villes. Au sein de Soyouzkniga existait aussi le "réseau des collecteurs de bibliothèques ", fondé en 1918 par Nadedja Kroupskaïa, et destiné à compléter les fonds des bibliothèques de tout le pays (il y avait 350000 bibliothèques en URSS et 200000 pour la seule Russie). Parallèlement avaient été mis en place le réseau de diffusion du parti communiste et le réseau académique, qui fonctionnaient comme des clubs réservés aux dirigeants de haut niveau ou aux académiciens : les destinataires avaient un accès privilégié, voire gratuit, aux publications. Il existait en outre un système d'abonnements pour certaines publications d'ouvrages classiques. Pour s'abonner, il fallait payer l'équivalent du prix d'un livre, soit une somme assez faible; le détenteur de l'abonnement pouvait venir ensuite acquérir les livres dans une librairie dépendant de la maison d'édition. Toutefois ces abonnements étaient en nombre limité, ce qui entraînait des pratiques de gestion de la pénurie. Un institut universitaire, par exemple, avait droit à quelques abonnements et les membres du personnel tiraient alors à la courte paille les heureux bénéficiaires (ce qu'on appelait le principe de la "loterie"). Les simples particuliers, dès qu'un nouvel abonnement était annoncé, venaient faire la queue devant la librairie pour avoir le droit de s'inscrire. L'engouement pour le livre était assez fort pour que les queues commencent dès la nuit précédant l'ouverture et que la milice à cheval ait parfois à intervenir pour rétablir le calme. Il était également possible aux particuliers et aux organismes d'obtenir des ouvrages par le réseau "Le livre par la poste". Cet organisme éditait des catalogues et recevait des commandes livrées par le service du courrier avec paiement à réception. Avait été également mis en place, depuis le milieu des années 1970, un étonnant système d'échange entre du papier récupéré et des bons donnant droit à des livres ${ }^{2}$. Sous l'invocation de principes écologiques, vingt kilos de papier permettaient d'acquérir un livre. Les aberrations du système soviétique se perçoivent bien là : les livres promis aux collecteurs de vieux papiers, et dits rares - par conséquent précieux - faisaient du coup l'objet de tirages spéciaux à des centaines de milliers, voire des millions d'exemplaires. Pour l'exportation de livres en direction des pays socialistes ou occidentaux avait été créé, en 1923, le système Mejkniga qui diffusait des livres russes en langue originale ou en traduction (plus de cinquante langues de traduction). Quatre maisons d'édition (Progress, Mir, Raduga, Russky jasik) avaient en effet pour mission de produire des livres en langues étrangères. De nombreuses librairies assuraient la vente de livres dans les villes: il en existait 220 à Moscou, avec 800 succursales dans la région. Enfin, une Foire internationale du livre se tenait chaque année à l'occasion de l'Exposition des performances techniques et économiques de l'URSS (VDNKH). Les livres étaient exposés, mais il n'y avait pas de vente directe aux particuliers; la Foire avait pour fonction de préparer les commandes passées par les organismes professionnels (bibliothèques, librairies, diffuseurs).

Les productions culturelles, selon les objectifs politiques proclamés, devaient être accessibles à tous; en conséquence, le prix des volumes était fixé à un niveau faible. Et, pour preuve de la supériorité du régime, l'URSS se targuait d'être le plus gros producteur mondial de livres. Les librairies regorgeaient d'ouvrages. Cependant, le marché du livre était dans un état de pénurie chronique, en raison d'une disjonction entre les attentes du public et la politique de planification. En vertu d'une conception des rapports sociaux qui attribuait aux seuls dirigeants la capacité de savoir ce qu'il convenait de faire pour le bien du peuple, la planification de la production était exclusivement déterminée par les décisions "d'en haut ". C'est ce que proclamait

2 - Voir Alekseï Levinson, "Papier récupéré et livres, offre et clemande dans une branche actuelle du commerce du livre ", in Alexandre Stroev (sous la dir. de), Livre et lecture en Russie, IMEC Éditions-Éditions de la Maison des sciences de l'homme, coll. "In Octavo", 1996, 312 p. 
un slogan affiché dans la Bibliothèque d'État de littérature étrangère de Moscou : "Il faut donner au lecteur le livre dont il a vraiment besoin et non pas celui qu'il demande. " La volonté de déterminer la consommation par une production définie et contrôlée par le pouvoir central entraînait en fait la conjugaison d'une offre massive de livres et d'un rationnement général.

Les ouvres des dirigeants du parti ou de partis communistes étrangers étaient éditées à des tirages extrêmement élevés, de même que la littérature nationale "classique" étudiée à l'école. C'est ainsi qu'entre 1948 et 1988 Pouchkine a été publié en URSS 1999 fois, avec un tirage cumulé de 340 millions d'exemplaires (pour une population d'environ 250 millions d'habitants). Durant la même période Gorki était édité 2034 fois, avec un tirage cumulé de 198 millions d'exemplaires, Tolstöi culminant à 2199 éditions et 409 millions d'exemplaires. Pour la seule période 1981-1985, Pouchkine a atteint 276 éditions (plus de 100 millions d'exemplaires), Tolstoï 258 éditions et 63 millions d'exemplaires $^{3}$. Les productions des membres de l'Union des écrivains bénéficiaient également d'un privilège éditorial. Ces derniers avaient un droit à publication régulière, les honoraires des publications venant s'ajouter à leur salaire fixe. En revanche, les auteurs contemporains ne bénéficiant pas des mêmes protections étaient édités - lorsqu'ils l'étaient - à des tirages insuffisants pour la demande.

La planification de l'édition et le fonctionnement des imprimeries avaient aussi pour conséquence un recours sélectif aux retirages lorsqu'un titre était épuisé. On estime qu'en $198860 \%$ des imprimeries fonctionnaient avec des machines vieilles de soixante ans et la plupart des entreprises d'impression, obéissant aux seules directives de leur administration centrale, jugeaient sans intérêt de garder "sur métal " la composition d'un ouvrage, alors même qu'il n'existait pas d'autre stockage, plus moderne, de composition de l'ouvrage ${ }^{4}$. Les rééditions concernaient surtout les manuels, la littérature classique et les livres de référence, alors qu'il n'y avait généralement pas de retirage pour la littérature moderne. Cela entraînait des pratiques consommatrices caractéristiques d'un marché de pénurie (achat par un client de plusieurs exemplaires d'un ouvrage intéressant nouvellement paru afin de les répartir dans son entourage). Le manque de diversité des publications, la non-adéquation, aléatoire ou voulue, entre les tirages et les possibilités de vente engendraient un sentiment général de frustration et un circuit officieux du livre. Dans un contexte d'abondance de livres, de librairies et de bibliothèques, l'accession aux publications faisant l'objet d'une forte demande du public relevait du privilège de position et de l'inégalité des ressources en matière de capital social. L'insuffisance quantitative des livres effectivement demandés dans les librairies nécessitait, pour l'accès à la marchandise, de disposer de "relations " parmi le personnel; la situation était similaire dans les bibliothèques publiques qui, soit ne disposaient pas de ces ouvrages, soit les avaient en quantité insuffisante (il fallait s'inscrire, parfois dès avant la parution, sur une liste d'attente souvent fort longue). Il n'était pas rare que les bibliothécaires ne missent pas en accès au prêt certains ouvrages très demandés, dont la circulation était réservée à leur entourage. Par ailleurs, les dépôts de librairie et les rayons des bibliothèques publiques étaient encombrés d'ouvrages jamais demandés. Un marché noir important s'était développé, où les ouvrages pouvaient être acquis pour une somme excédant largement leur prix officiel. Alimenté par des détournements d'ouvrages depuis les imprimeries, ou bien à l'occasion du transport et de l'arrivée dans les librairies, il concernait aussi bien des auteurs russes qu'étrangers, des ouvrages littéraires que des publications scientifiques. Il y avait de ce fait au sein du marché noir des livres des sousmarchés spécialisés (romans historiques, policiers, science-fiction, poésie, philosophie). Les vendeurs du marché noir (en russe "speculants ") se trouvaient dans des lieux publics, à proximité des grandes librairies ou sur certaines places. La police tenait ce marché sous contrôle en limitant l'entrée de nouveaux vendeurs, en surveillant les livres échangés et en prenant éventuellement une part des profits dégagés. Le réseau du "Samizdat " diffusait clandestinement des textes n'ayant aucune chance de publication en URSS en raison de leur contenu idéologique ou de leur origine: leur circulation passait par des copies dactylographiées, des photocopies ou même des photos page à page de livres publiés en Occident. C'est encore sous cette forme que l'œuvre de Nietzsche, par exemple, était accessible au début des années $1980^{5}$. Le public du "Samizdat " était composé principalement d'universi-

\footnotetext{
3 - Voir Lev Goudkov et Boris Doubine, "La culture littéraire, ration et production ", in Alexandre Stroev, op. cit.

4 - Voir Lev Goudkov et Boris Doubine, art. cit.

5 - Les ceuvres de Nietzsche en langue originale ou en traduction russe éditées avant la révolution bolchevique existaient en bibliothèque, mais leur accès était réservé à des spécialistes munis d'autorisations de consultation émises par les autorités universitaires.
} 
taires, d'intellectuels ou d'étudiants. Enfin, outre la censure idéologique, abondamment dénoncée en Occident, était exercée une censure éthico-esthétique. La littérature "bas de gamme "(romans populaires sentimentaux, policiers, thrillers) était jugée néfaste pour les lecteurs et indigne de publication, à de rares exceptions près. Il existait bien une "littérature de masse ", productrice de romans édités parfois à des millions d'exemplaires, et beaucoup lus, mais elle s'inscrivait dans une perspective éducative: absence des thèmes tabous (violence, érotisme), récits de type "réaliste socialiste" exaltant des idéaux humanitaires, patriotiques ou socialistes, écriture "correcte" évitant les termes argotiques.

Les ouvrages du marché noir et du "Samizdat " donnaient donc une bonne indication des déficits de l'édition soviétique. Dès que le monopole d'État sur la production et la distribution a été aboli et la censure assouplie, des entrepreneurs ont pu investir ce créneau : parmi eux d'anciens vendeurs du marché noir ayant acquis par leur activité illégale de l'argent investissable et une fine connaissance de la demande. Des profits énormes ont été réalisés. Pendant quelques années, le commerce de livres a été en Russie l'un des plus rentables, tout juste dépassé par celui de la vodka.

\section{MULTIPLICATION DES ÉDITIONS PRIVÉES ET RESTRICTION DU MARCHÉ}

Les premières créations d'entreprises privées, sous forme de coopératives, ont eu lieu en 1988, ce qui a eu pour effet immédiat une visibilité nouvelle des ouvrages et un bouleversement de la production. Le tournant de la décennie 1980-1990 a pu être vécu par le public comme une fête de la lecture, inattendue et prolongée. Les livres sont sortis dans les rues, les carrefours, les entrées de métro. De même que se multipliaient les kiosques de rue vendant pêle-mêle des alcools occidentaux, de la vaisselle et de la lingerie, de même se multipliaient les étals proposant au passant la juxtaposition bariolée de traductions d'ouvrages de la collection "Harlequin", de grands textes littéraires, d'essais politiques et de calendriers astraux. Les maisons d'État, du coup, ont été amenées à redéfinir leur programme éditorial et à prendre en considération, au moins partiellement, le critère de rentabilité et peut-être plus encore les changements de rapports de force politiques. C'est ainsi que le livre de Berdiaev Destin de la Russie, publié initialement par une maison coopérative avec un petit tirage et une médiocre qualité typographique, a été rapidement épuisé et quasiment aussitôt republié par la prestigieuse maison d'État Naouka à 100000 exemplaires. Peu après, les Presses universitaires de Moscou republiaient l'ouvrage, concurremment à une réédition par Naouka. Il est encore plus remarquable que les ouvrages de Zinoviev, interdits de publication en URSS jusqu'en 1989, aient été publiés en 1991 par la maison d'édition qui appartenait encore à cette époque au comité du parti communiste de Moscou. Les livres précédemment victimes de la censure politique ont fait, au début de la décennie, de très grosses ventes, cependant qu'apparaissaient de nouvelles catégories d'ouvrages. Les observateurs ont souligné la soudaine présence des ouvrages érotiques dans les pays de l'Est, qui contrastait avec la pruderie antérieure. Le développement considérable des romans d'amour "pour femmes ", des romans policiers, des récits d'aventure ou de sciencefiction montre bien qu'il existait une demande latente de littérature populaire, non satisfaite au nom d'impératifs de valeur culturelle et éducatrice des textes. Les bénéfices tirés de la vente rapide de ces ouvrages étaient tels que des commerçants ayant installé des stands de rue achetaient d'avance à l'éditeur la totalité du tirage pour en avoir l'exclusivité.

Pendant quelque temps, l'édition et la vente de livres ont été des secteurs où pouvaient se jouer, avec une mise initiale faible, de grosses opérations. Et aussi, du fait des sommes en jeu et de l'apparition rapide de groupes criminels dans la vie économique, un domaine où tous les coups étaient permis : éditions pirates en quantité, attaques à main armée de locaux, rackets mafieux, détournements de livres à partir de l'imprimerie ou du transport. Avec une concurrence sauvage entre éditeurs les amenant à tenir secrètes leurs futures parutions et à rivaliser de vitesse avec leurs pairs. Les traductions de best-sellers occidentaux ou de romans populaires étaient effectuées en toute hâte; parfois même l'éditeur se contentait de déchirer un volume et de répartir les pages entre des traducteurs improvisés chargés essentiellement de faire vite et d'être discrets. Les possibilités de profits rapides expliquent en partie la multiplication des entreprises. En 1993, l'administration russe enregistrait déjà 6000 entreprises ayant licence d'édition et 7000 en 1995. Mais beaucoup d'entre elles n'ont existé que sur le papier et nombre de celles qui ont effectivement publié des ouvrages n'ont eu qu'une activité éphémère ou sporadique. La disjonction entre les chiffres officiels et la réalité du secteur éditorial tient à plusieurs facteurs. L'acquisition d'une licence 
d'édition était une démarche assez simple et peu coûteuse. Des entrepreneurs potentiels ont saisi cette opportunité, sans avoir véritablement décidé du domaine dans lequel ils voulaient et pouvaient s'engager. D'autres ont publié quelques ouvrages, avec ou sans succès, avant de s'arrêter provisoirement ou définitivement. La cessation d'activité d'une maison ne signifiait pas toujours que ses propriétaires avaient quitté le domaine de l'édition. Les lacunes de la législation ont permis pendant un moment à certains de se soustraire à leurs créanciers en déclarant en faillite leur première entreprise, recréée sous un autre nom, avec le même personnel et le même matériel. La multiplication de petites entreprises pouvait aussi se révéler une efficace adaptation à la politique fiscale ${ }^{6}$. Les maisons éphémères correspondaient aussi parfois à des stratégies d'accumulation de capital économique associées à un souci de préservation d'une image de marque. Le dirigeant d'une maison d'édition ayant pour ambition de publier des livres de qualité, mais à faible rentabilité, ouvrait une autre maison dévolue à la publication d'ouvrages générateurs de gros profits, réinvestis ensuite dans la maison "noble "; l'opération pouvait être réitérée avec des maisons annexes suivant l'évolution des ouvrages à grosse vente.

C'est l'apparition de petites entreprises concurrentielles qui a modifié profondément le secteur éditorial russe, beaucoup plus que la privatisation des anciennes maisons de l'époque soviétique. Dès lors que celles-ci ont perdu à la fois leur situation de monopole et le soutien financier de l'organisme auquel elles appartenaient, qui se désengageait ou disparaissait, elles sont apparues comme des dinosaures, inadaptées à la nouvelle situation, entravées par leur personnel pléthorique et les lourdes infrastructures dont elles ne pouvaient plus assumer le coût. Une partie de leurs dirigeants sont partis créer de nouvelles entreprises, réinvestissant sur le marché libre les compétences et le capital social acquis antérieurement.

"Un de mes anciens collègues est directeur d'une maison d'édition connue. Ils ont des collections comme "Bibliothèque des romans pour les femmes", "Caprice", "Passion", une collection de romans populaires. Pourquoi est-ce que mon collègue a quitté son poste ici? Parce qu'ici il ne pouvait pas faire de profit. Avec l'arrivée des rapports capitalistes, les maisons d'édition ne rapportent plus beaucoup d'argent. Nous avons ici une très riche bibliothèque, des œuvres complètes, des livres anciens : ça coûte cher. Et que voulaient les jeunes? Ils cherchaient le moyen de s'enrichir vite. Si on paie des gens travaillant dans la bibliothèque, le profit diminue. Mais si je quitte cette maison d'édition, si je prends seulement trois ou quatre personnes avec moi, je reçois de l'argent en liquide et je deviens millionnaire. Voilà... En plus, les gens qui ont quitté les maisons d'édition ont utilisé ce qu'ils y avaient appris. À l'époque, le rédacteur en chef de notre maison pouvait ouvrir toutes les portes. Il visitait toutes les foires du livre à l'étranger, où il pouvait acheter des droits d'auteur sans problèmes, et ça coûtait très peu. Il a donc pu utiliser ça pour ses propres affaires. Et puis il connaissait les auteurs; c'est un capital très important. " (Rédacteur en chef d'une ancienne maison d'édition d'État, privatisée et continuant difficilement son activité ; entretien réalisé en 1996.)

La possession de locaux bien situés a permis toutefois à ces anciennes maisons de disposer d'une source de financement, puisqu'elles ont pu en louer une partie à des entreprises privées d'autres secteurs. Si le nombre de titres publiés et les tirages ont été très fortement réduits, certaines maintiennent une activité soit en jouant la carte de la production de masse (littérature populaire), soit en visant étroitement un public professionnel spécialisé (livres scientifiques, médicaux).

En 1998, près de 11000 maisons d'édition étaient enregistrées. Les licences ayant été accordées pour cinq ans, une baisse forte était prévue à courte échéance, après élimination des maisons fantômes créées en 1993-1994 (le président du Comité pour la presse de Russie estimait, à l'automne 1998, que $40 \%$ environ des entreprises déclarées avaient une activité).

Créer une petite entreprise d'édition ne nécessite pas un capital économique initial important. Nombre de maisons fondées au début de la décennie ont été constituées de manière tout à fait artisanale: elles étaient installées au domicile du propriétaire, le personnel se réduisant à sa famille et l'équipement à quelques ordinateurs. Certains éditeurs ont pu trouver un premier financement auprès d'individus ayant déjà eu la possibilité d'accumuler du capital sur l'ancien marché noir ou sur le nouveau marché libre. Le transfert de la propriété d'État à la propriété privée n'ayant été ni préparé, ni contrôlé, il a été possible parfois de tirer parti de la situation pour acquérir des infrastructures ou des fonds. Les nouveaux éditeurs sont en général d'une très grande discrétion sur les conditions matérielles de fondation de leur entreprise et sur ses financements initiaux. Le recours à l'emprunt bancaire a été et reste toujours rare, compte tenu des taux élevés et des délais de remboursement extrêmement brefs. Les

6 - Au début de la décennie, les entreprises devaient payer une taxe sur les salaires versés à leurs employés dès que les rémunérations excédaient un certain seuil. Il était de ce fait avantageux de répartir le salaire versé à un employé entre plusieurs entreprises. 


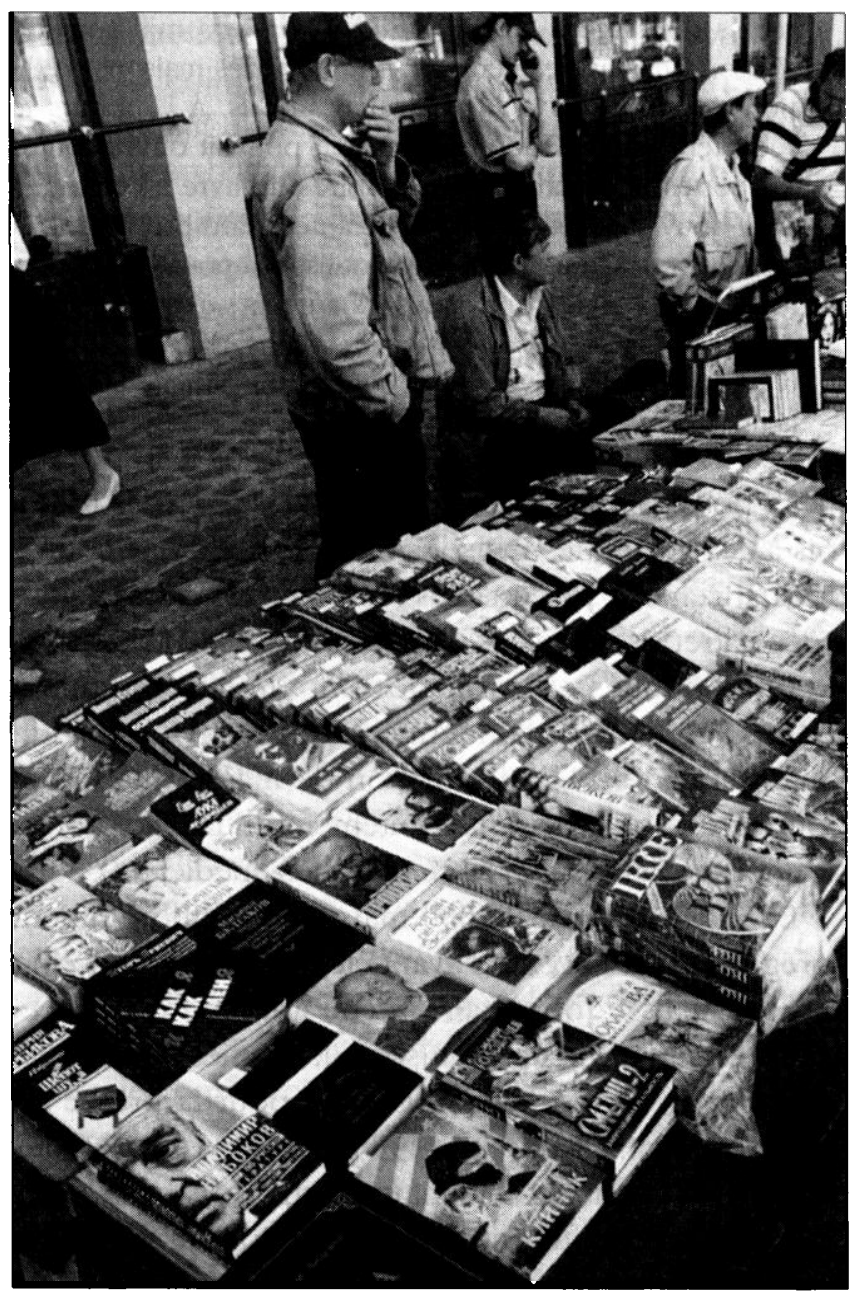

Moscou. Vente de livres dans la rue. Mélange de livres religieux, de cuisine, de philosophie, de romans populaires, d'œurres de Lénine et de Trotsky.

entreprises ne peuvent compter que sur leurs profits pour réaliser les investissements nécessaires à leurs activités, sauf dans les quelques cas où une maison d'édition est une des filiales d'un groupe ayant des activités multiples - ou bien sert à couvrir, par de pseudodroits d'auteur versés à des hommes politiques, des pots-de-vin. Au début de la décennie, l'inflation énorme a obligé les éditeurs à produire des ouvrages vendables en quelques semaines, chaque jour passé laminant les bénéfices escomptés. Même si le taux d'inflation a sérieusement diminué, l'écoulement rapide reste un impératif pour la survie des entreprises puisqu'elle détermine la possibilité de placer les ouvrages sur les lieux de vente.

L'élément essentiel actuellement pour l'édition russe est l'absence de véritables organismes de distribution. Les anciennes structures, dès qu'elles ont cessé d'être subventionnées par l'État, se sont trouvées submergées de dettes et incapables de fonctionner. Là encore des entreprises privées à faibles effectifs se sont créées. Cela va de l'individu qui, avec son véhicule, va chercher des ouvrages directement chez l'éditeur et les place sur des points de vente, jusqu'aux grossistes qui disposent d'entrepôts. Le haut lieu de la distribution de livres à Moscou est le Stade olympique construit pour les Jeux de 1980. En 1992, des individus enrichis dans le marché noir ont utilisé les coursives du stade désaffecté pour y installer des stands destinés à alimenter pendant le week-end les vendeurs sur étal moscovites. Cette initiative d'abord illégale et marginale s'est prolongée et organisée. Une société par actions a été constituée, qui a en bail les soubassements du stade et les a transformés en centre commercial. On y trouve actuellement un grand marché du livre, de gros et de détail. Des sous-locations, par contrats à brève durée - six mois maximum -, sont accordées par la société gérante à des marchands qui tiennent des éventaires sur des tréteaux ou à même le sol. Ils peuvent éventuellement disposer de réduits pour stocker leur marchandise. Le marché du livre est désormais ouvert cinq jours par semaine et, moyennant un droit d'entrée modique, particuliers et marchands peuvent venir s'approvisionner. La foule qui se presse au Stade olympique est extrêmement dense et les conditions d'hygiène et de sécurité effrayantes. D'énormes quantités d'argent liquide circulent, qui échappent au contrôle du fisc. Le chiffre d'affaires moyen d'un sous-locataire de stand est de mille dollars par jour. C'est un service d'ordre interne, qui s'occupe des conflits éventuels. Des détaillants emportent des ouvrages pour les revendre à Moscou ou en province, les modes de transport allant de la simple carriole ou poussette aménagée jusqu'au camion. Si la diffusion de livres peut se maintenir par ce type d'expédients, la situation pose de sérieux problèmes aux éditeurs. Ils sont encombrés par leurs stocks de livres que les divers diffuseurs viennent chercher au fur et à mesure, en fonction des ventes escomptées à court terme. L'expérience incite les éditeurs à la méfiance envers les distributeurs qui paient le plus lentement possible, et parfois ne paient pas. Les livres à vente lente, et notamment les ouvrages scientifiques, trouvent difficilement preneur. Les éditeurs de livres intellectuels doivent prendre une part active dans la commercialisation de leur production.

"Dans la majeure partie des cas, les livres intellectuels se vendent "à la main", c'est-à-dire que l'éditeur s'occupe luimême de la diffusion en contactant les librairies et ce sont 
les employés de l'éditeur qui expédient les livres chez le libraire. Souvent, des petits grossistes et des libraires viennent directement à la maison d'édition pour acheter une petite partie du tirage et ils les emportent pour les vendre à Moscou et à Saint-Pétersbourg. Aujourd'hui, il existe plusieurs prix pour le même livre. Le premier prix, c'est lorsque le grossiste paie immédiatement la totalité des livres achetés, et qu'on lui fait alors une remise. Il y a un deuxième prix quand on le vend à un grossiste ou à un libraire fiable qui va payer au fur et à mesure. Et un troisième prix quand on vend à un intermédiaire dont on n'est pas sûr qu'il arrivera à vendre la totalité dans des délais convenables. Un éditeur risque toujours que l'argent des ventes faites par le libraire ou le grossiste ne lui soit pas retourné, ou bien qu'il soit payé au bout d'un an. " (Directeur d'une petite maison d'édition, spécialisée dans la philosophie contemporaine, entretien réalisé en 1996.)

Alors que les maisons d'édition, à l'époque soviétique, étaient déchargées par les organismes spécialisés de toutes les questions concernant l'écoulement des ouvrages, celles-ci sont devenues un élément essentiel de l'activité des entreprises. L'absence de sécurité des échanges commerciaux et la nécessité pour les éditeurs de s'occuper de la vente explique que les nouvelles maisons privées ayant l'ambition de durer essaient d'ouvrir des librairies à leur enseigne. Mais la propriété ou la location immobilière, dans la capitale, est devenue très coûteuse. La maison d'édition Ad Marginem - ce n'est pas la seule dans ce cas - a créé à Moscou une librairie dans la cave d'un immeuble d'habitation d'une rue non commerçante. En revanche, les éditions Terra, au catalogue fourni et diversifié, possèdent, signe de réussite et d'accumulation de capital, plusieurs librairies bien situées en centre-ville, de même que des imprimeries. Du fait de l'acheminement au coup par coup, les livres publiés à Moscou et à Saint-Pétersbourg n'arrivent que difficilement en province, et avec un prix fortement majoré par la série des intermédiaires et du transport. Or les salaires sont sensiblement inférieurs en province, ceux des employés des entreprises publiques étant de surcroît versés très irrégulièrement. Inversement, les ouvrages publiés en province n'arrivent que rarement à Moscou et à Saint-Pétersbourg. L'essentiel de la production est concentré dans les deux grandes villes ( $75 \%$ des titres et $80 \%$ des tirages) où s'effectue la majeure part de la consommation. Extrême réduction spatiale du marché... Dans la seconde moitié de la décennie ont été tenues, dans les grandes villes de province russes, des foires du livre qui n'étaient plus seulement des expositions de nouveautés, mais aussi des lieux de vente. Les éditeurs y proposaient directement leurs ouvrages, ce qui permettait d'éviter les sur- coûts des intermédiaires. Le retour immédiat de l'argent compensait amplement les frais de déplacement du personnel et la location de stands.

Le riche ensemble de librairies a de toute façon été mis à mal par la privatisation. À Moscou, cinquante librairies ont disparu pour faire place à d'autres commerces et beaucoup de celles qui restent ont dû, pour maintenir leur équilibre financier, louer une partie de leurs locaux à des vendeurs d'informatique, des stands de développement de photos ou des marchands de cosmétique. En revanche, beaucoup de librairies "de plein air ", à infrastructure légère (une planche sur deux tréteaux!), sont apparues à proximité des anciennes, certaines rues étant quasiment spécialisées dans ce genre de commerce. La question de la diffusion des livres est assez cruciale pour que le responsable d'une nouvelle maison d'édition, interviewé lors de l'étude, refuse de répondre à la question en arguant du " secret commercial". Un projet lancé en 1998 prévoyait la reconstitution d'un organisme central de diffusion et de distribution, au niveau de la ville de Moscou. Une importante société initialement spécialisée dans le commerce alimentaire et la restauration et qui s'était engagée récemment sur le marché du livre s'apprêtait à reprendre les vingt plus grandes librairies de la ville et à contrôler la distribution, en contrepartie d'un paiement à la mairie de Moscou d'impôts supérieurs à ceux réglés par les petites sociétés jusque-là locataires des locaux. Le caractère anarchique de la diffusion et de la distribution de livres est dénoncé par tous les éditeurs comme un obstacle à leur activité, mais la mise en place d'un quasi-monopole peut entraîner la disparition de beaucoup d'entre eux, qui n'auront plus la ressource de traiter directement avec les librairies.

\section{LES MODIFICATIONS DE LA PRODUCTION}

Si les tirages, jusqu'à la fin de la perestroïka, ont gardé une ampleur "soviétique " (la centaine de milliers d'exemplaires, pour une première édition, n'étant pas rare), ils ont très rapidement diminué. Ils ont été ajustés aux possibilités de vente à court terme et à la restriction de l'espace de vente, ce qui a eu pour effet une importante réduction du nombre de volumes publiés. Alors que le tirage total des livres, depuis 1980, avait constamment dépassé 1,300 milliard de volumes par an, et avait culminé à 1,800 milliard en 1988, il est tombé à moins de 600 millions en 1994 et à 422 millions en 1996 (il est d'un peu moins de 400 millions en France). 
"En 1989-1990, nous avons édité notre Dictionnaire de l'Antiquité, tiré à 100000 exemplaires. L'édition a été épuisée en un clin d'oeil dans tout le pays (c'était encore l'URSS). Je ne sais pas si cette édition est parvenue en province, le tirage a été beaucoup trop vite écoulé. En 1992-1993, le dictionnaire a été réédité avec un tirage de 50000 exemplaires, lui aussi vite épuisé. Mais déjà un an plus tard les tirages à 25000 commençaient à traîner en magasin. Je crois que ça s'explique par l'augmentation des prix et aussi parce que d'autres maisons d'édition ont surgi. Avec la nouvelle liberté, chaque maison d'édition s'est ruée sur le marché en commençant par éditer ce que l'acheteur voulait avoir dans sa propre bibliothèque. Actuellement, un tirage à 10000 est déjà considéré comme élevé. Nos livres sortent en 3000 à 5000 exemplaires parce que ce sont des publications scientifiques." (Rédacteur en chef des Éditions du Progrès, ancienne maison d'État, entretien réalisé en 1997.)

Les disparités sont fortes selon les genres d'ouvrages : il y a eu des tirages, vite épuisés, de plusieurs centaines de milliers d'exemplaires pour des ouvrages interdits à l'époque soviétique, et les romans populaires peuvent encore atteindre des chiffres impressionnants, tandis que les ouvrages "intellectuels " ne sont édités généralement qu'à quelques milliers d'exemplaires. Ce qui correspond à la situation de l'édition occidentale, mais ne laisse encore pas de surprendre en Russie. Le nombre de titres publiés s'est effondré dans un premier temps - en 1993, il était tombé au niveau des années 1930 -, toutefois il a regagné, dès 1996, son niveau de 1990. Là encore, la situation s'est trouvée très contrastée selon les genres. En nombre de titres, les fictions romanesques ont connu une augmentation certaine : 4676 titres publiés en 1980 et 6792 en 1994, ce dernier chiffre étant probablement inférieur à la réalité puisque le dépôt légal, particulièrement pour la littérature populaire, n'a pas été rigoureusement respecté. Des genres se sont considérablement développés, à partir de rien ou presque : religion, ésotérisme, astrologie. Le secteur des livres pratiques et de "l'art de vivre" a connu un essor important, particulièrement dans les domaines négligés à l'époque soviétique: médecine par les plantes, cuisine, décoration ${ }^{7}$. Parmi les nouveaux genres de publications figurent les ouvrages relatifs aux nouvelles conditions de la vie économique et les manuels de management. Les encyclopédies ont connu un gros succès, en raison du déficit antérieur d'ouvrages de vulgarisation correctement illustrés et du sentiment largement répandu dans la population d'avoir été longuement privé du "vrai " savoir, ou d'avoir été victime de l'inculcation de faux savoirs. Les livres pour enfants, notamment par la traduction de publications occidentales, ont connu une forte expansion qui va de pair avec le changement des autres produits destinés à l'enfance (fort engouement pour les jouets occidentaux notamment - en Russie, le Journal de Barbie tirait, en 1996, à un million d'exemplaires...). En période de crise et de renouvellement des références, les parents réservent une part non négligeable de leur budget, au détriment sans doute de leur propre consommation, à la nouvelle génération afin de lui permettre, autant que faire se peut, d'accéder aux consommations enviées. Les changements dans les programmes scolaires ont entraîné une croissance forte dans le secteur des manuels. Jusqu'en 1994, ils étaient tous distribués gratuitement aux élèves. Une partie est maintenant à la charge des familles. Les entreprises qui se sont lancées dans ce sous-secteur à public nombreux et captif - l'une d'entre elles a été montée par d'anciens dirigeants de la maison d'État spécialisée - ont été confrontées à une intense concurrence pour obtenir les commandes d'État - avec ou sans subvention - ou le label indiquant que l'ouvrage est recommandé par le ministère de l'Éducation. En 1996, le directeur, le directeur adjoint ainsi que le chef comptable de l'une des entreprises concernées ont été assassinés. Les publications scientifiques sont en revanche en fort déclin, la baisse traduisant les effets conjugués du désengagement de l'État et des difficultés budgétaires que rencontrent les universités et les bibliothèques.

Une part extrêmement importante des publications russes, au début de la décennie, a été composée de traductions. Les romans d'amour, les policiers, les thrillers qui avaient fait défaut durant la période soviétique sont arrivés d'abord par des œuvres occidentales: la série des Angélique (connue jusque-là par des films interdits aux moins de seize ans), les enquêtes d'Hercule Poirot, la collection "Harlequin" et la science-fiction américaine ont fait les premiers gros coups éditoriaux du genre. En 1992, 78\% des volumes relevant de la catégorie fiction-poésie étaient des traductions, mais ce chiffre était déjà descendu à 60\% en 1994 et à 47\% en 1996. Une production autochtone de romans populaires, sur le modèle occidental, est en effet vite apparue et les éditeurs de ce type de littérature ont rapidement constitué des "écuries" d'auteurs spécialisés. Les traductions

\footnotetext{
7 - Le genre nouveau des guides de voyage, destinés à la fraction de la population assez fortunée pour voyager à l'étranger, a connu également un développement certain. Une des rares implantations étrangères dans le domaine du livre est la librairie ouverte dans le centreville de Moscou par l'entreprise française éditrice du Petit Fâté. Elle vend des cartes et de la documentation touristique.
} 
tiennent évidemment une place essentielle clans tous les sous-secteurs inexistants à lère soviétique (livres de management, art de vivre). Les publications religieuses, interdites à l'époque soviétique, se sont développées à partir de rééditions d'ouvrages orthodoxes antérieurs à la révolution ou d'auteurs russes en exil, mais aussi de traductions ( $L a$ Vie de Jésus de Renan a été éditée douze fois au début de la décennie). Les traductions sont restées dominantes dans des secteurs comme lès sciences sociales ou la philosophie. La déstabilisation des spécialistes russes, qui ont perdu d'un coup la base intellectuelle de leurs réflexions et de leurs recherches et qui écrivent beaucoup moins, se conjugue avec l'engouement du public pour les auteurs occidentaux longtemps interdits. Les chercheurs et les universitaires qui, sous le régime soviétique, pouvaient faire publier leurs livres grâce aux plans de production établis dans les maisons d'état ont perdu leurs débouchés avec le passage au marché. Ils doivent maintenant assurer eux-mêmes le financement de leurs publications, soit sur fonds propre, soit par une subvention. L'essentiel des subventions en ce domaine est apporté par des fondations ou des organismes occidentaux qui soutiennent à peu près exclusivement les traductions d'auteurs étrangers.

À l'époque soviétique, la traduction était considérée comme une activité prestigieuse. Elle ćtait exercée par des professionnels spécialisés, passés par les très sélectives écoles de traducteur-interprète, ou par des universitaires ou écrivains ayant une bonne connaissance d'une langue étrangère. Le travail de traduction poldvait impliquer la rédaction d'un appareil critique de l'ceuvre. Bien rémunérée, cette activité a pu servir de refuge à des créateurs dont les propres ceuvres rencontraient des difficultés à la publication (Boulgakov, Pasternak). L'augmentation importante des traductions et la part prise par les ouvrages "sans valeur culturelle " ont fait surgir de nouveaux traclucteurs aux compétences norl garanties, mais acceptant de travailler rapidement et à faible coût.

Par comparaison avec l'époque soviétique, le livre est devenu une marchandise onéreuse: un volume relevant de la "production de masse " coûtait, en 1998, l'équivalent de 10 à $20 \mathrm{~F}$ (le salaire d'un ouvrier ou d'un employé dans la capitale était, au premier semestre 1998 , de 1500 à 2000 F alors que Moscou est devenue une ville où le coût de la vie est l'un des plus élevés au monde). Les livres "intellectuels "sont sensiblement plus chers, leurs prix correspondant à 30 ou $50 \mathrm{~F}$. Cette majoration s'explique en partie par la persistance d'une association, héritée de l'époque soviétique, entre ouvrage de qualité et couverture "dure". Alors que la

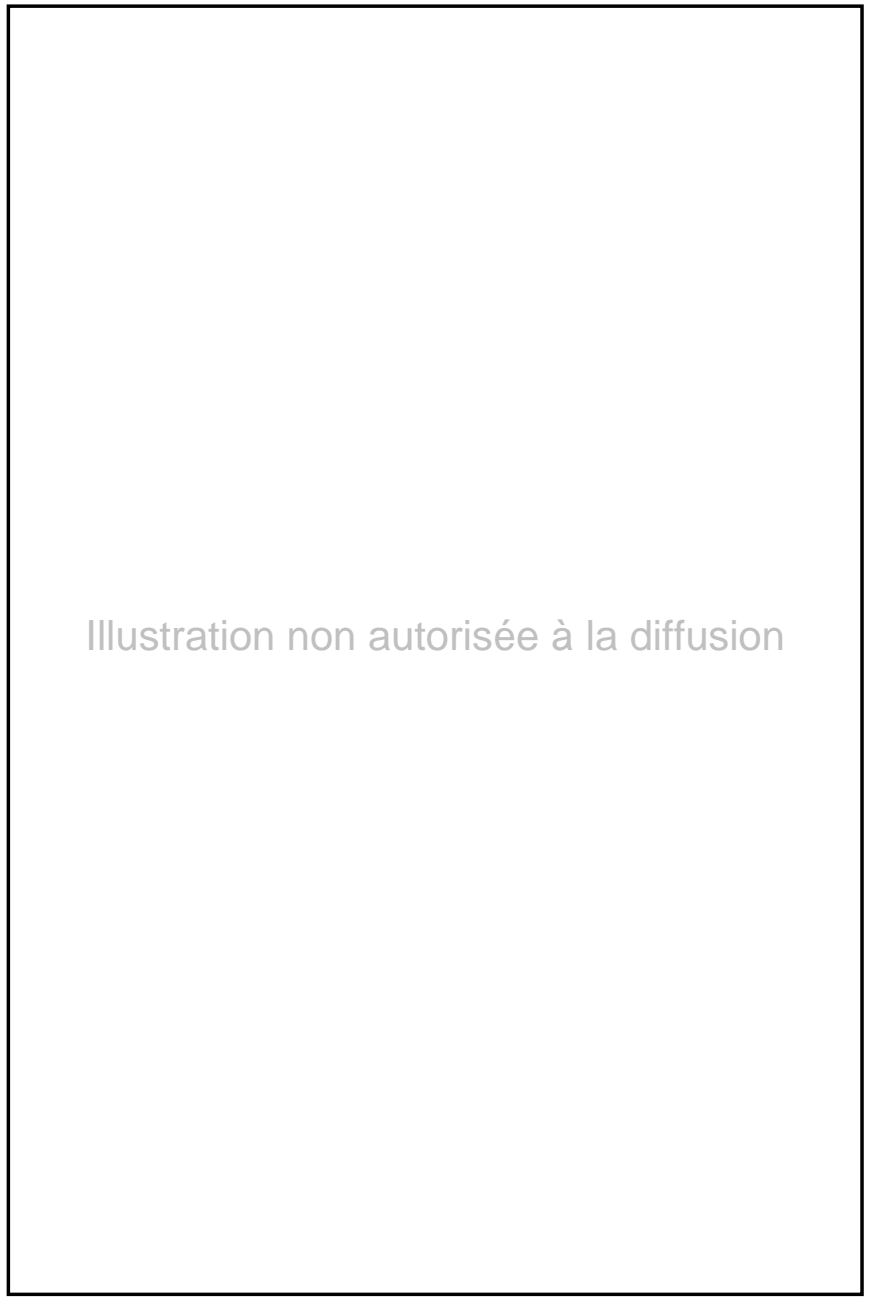

Le modèle anglo-saxon de couverture, avec lettres dorées ou en relief, s'est imposé pour le roman populaire.

production de masse adopte souvent le modèle américain de présentation des livres populaires (couverture souple, imagée et très colorée, avec un titre en lettres dorées ou argentées), la production de qualité doit encore fournir des couvertures cartonnées, de fabrication plus coûteuse. Dans le même temps, les salaires des intellectuels sont particulièrement faibles (un professeur d'université gagnait, au premier semestre 1998, de 800 à $1000 \mathrm{~F}$ par mois). On estime qu'au début des années 1980, le salaire d'un chercheur débutant équivalait à une centaine de livres, mais ne correspondait, début 1998, qu'à 15 ou 20 livres. Néanmoins, persistance de comportements hérités de l'époque soviétique, l'achat de livres était, jusqu'en 1998, encore relativement élevé en Russie compte tenu des difficultés matérielles auxquelles la majeure partie de la population était confrontée. Une étude menée en 1997 a noté que $23 \%$ des enquêtés avaient acheté au moins un livre 
pendant les douze mois précédents, la proportion étant nettement plus élevếe à Moscou où les titulaires d'un diplôme de l'enseignement supérieur représentent 34\% de la population : $9 \%$ des enquêtés (et $12,5 \%$ des titulaires d'un diplôme universitaire) avaient acheté au moins un livre par mois. Cependant la crise de l'été 1998 a fortement affecté le secteur du livre. Enchérissement brutal des produits de première nécessité, importante baisse du pouvoir d'achat de la plus grande partie de la population, retards de versement des salaires : les vendeurs de livres ont estimé qu'ils ne pouvaient augmenter sensiblement les prix sans perdre l'essentiel de leur clientèle.

\section{UN LIEU DE RECONVERSION}

Alors que, dans le système soviétique, les responsables et les personnels de haut niveau des maisons d'édition étaient parfaitement spécialisés et avaient eu un type de formation précis, la privatisation a ouvert le secteur à des individus qui n'y avaient jamais travaillé. Parmi eux, des gens à faible capital culturel qui se sont lancés dans la publication de livres dans la mesure où il s'agissait d'une marchandise susceptible de procurer de forts profits. Ils se sont occupés de livres comme ils ont fait, avant ou après, de l'alimentation ou de l'électronique. Mais la plupart des responsables de maisons d'édition ayant une activité durable sont fortement diplômés. Ce sont souvent d'anciens cadres des maisons d'État, d'anciens universitaires ou journalistes. La création d'entreprises privées a été pour les premiers le moyen de continuer à utiliser leurs compétences professionnelles dans le nouveau contexte. Au prix de l'apprentissage d'un nouveau métier, les seconds ont tenté de pallier les pertes, tant symboliques qu'économiques, qu'entraîne pour eux la dévalorisation radicale des positions qu'ils occupaient antérieurement.

Les professions intellectuelles et scientifiques, à l'époque soviétique, n'étaient pas sensiblement mieux rétribuées que les professions manuelles ou techniques, mais elles étaient hautement considérées. Avec le changement, elles ont été confrontées à la fois aux difficultés matérielles communes aux employés du secteur public tout en perdant le prestige qui leur était conféré auparavant. L'État, qui n'attend plus des sciences et de la culture une légitimation, s'en est fortement désengagé. Les instituts universitaires ne disposent plus des moyens leur permettant de fonctionner décemment et les salaires de leur personnel ont été fortement réduits.
Un universitaire ne peut vivre à Moscou ou à SaintPétersbourg s'il n'ajoute à son traitement des revenus annexes. Dans le même temps, le discrédit attaché à l'ancien système et à son idéologie officielle atteint toutes les positions symboliquement valorisées auparavant. Les détenteurs de ressources culturelles importantes sont donc dans la nécessité de redéfinir leur place dans la société postcommuniste en inventant et en investissant de nouvelles positions. Deux stratégies principales ont été mises en œuvre dans cette conjoncture: soit l'abandon total du domaine scientifique et culturel et la reconversion dans le nouvel espace du pouvoir économique et politique, soit une démarche de transposition qui s'appuie sur le capital culturel et scolaire et essaie de le faire à nouveau fructifier en l'adaptant aux nouvelles conditions de la vie économique et sociale. Les universitaires ayant un savoir plus "empirique " peuvent éventuellement le faire travailler comme "science appliquée", répondant aux commandes du marché : les sociologues ont ainsi la ressource de faire du marketing ou des sondages, les économistes d'enseigner l'économie de marché dans les nouvelles écoles ou instituts qui se sont multipliés ces dernières années, les scientifiques de devenir consultants d'entreprise, experts en finances ou en informatique. Les professions antérieurement les plus "idéologiques " et les plus liées à l'ancien régime (philosophes, professeurs de marxisme-léninisme, journalistes) se réinsèrent surtout dans le nouvel espace politique et médiatique : appareils des nouveaux partis, chaînes privées de télévision, entreprises culturelles. On retrouve de fait un nombre non négligeable d'anciens philosophes universitaires ou de journalistes parmi les nouveaux dirigeants d'éditions privées, qu'elles soient spécialisées dans la production de masse ou dans la production intellectuelle.

\section{ÉduQuer le pUBLIC, S'AJUSTER AUX CONSOMMATEURS}

Dans le système soviétique, les destinataires des publications étaient perçus comme une masse à éduquer pour laquelle il fallait planifier des lectures saines et formatrices. L'édition était conçue comme un travail pédagogique et les rédacteurs des maisons d'État se considéraient comme des " archilecteurs" idéaux, intermédiaires entre créateurs et fonctionnaires du Comité d'État à l'édition, chargés de définir ce qui était bon et utile pour le public. La période de la perestroïka a 
d'ailleurs été vécue par ces rédacteurs comme un moment heureux, puisque l'affaiblissement du contrôle politique leur permettait de produire plus aisément les ouvrages qui leur plaisaient, tout en restant affranchis de toute obligation de rentabilité. L'abolition du monopole étatique, en revanche, a brusquement transformé le livre en marchandise. A disparu ce "public" qui devait s'accommoder de l'offre déterminée pour lui. Les éditeurs se sont trouvés face à une espèce nouvelle, les consommateurs. Le choc a été d'autant plus rude que la disparition de la censure éthique et esthétique a permis la production d'une littérature populaire aux ventes particulièrement élevées en raison du déficit antérieur en la matière. Partagés entre l'ancienne définition valorisante de la profession (faire des ouvrages de qualité pour former le public) et les contraintes économiques nouvelles (deviner les goûts d'un ensemble hétérogène de consommateurs pour réaliser des retours sur investissement à court terme), nombre d'éditeurs à fort capital culturel ont voulu penser que la production des " mauvais" livres était un détour inéluctable mais provisoire avant de revenir - dans la liberté idéologique - à la publication d'ouvrages de qualité. Dans les anciennes maisons d'État, la compromission avec la production de livres "bas de gamme" a été vécue comme une humiliation.

"On avait un ministre du Comité d'État de presse, c'est aujourd'hui de mauvais ton de dire que c'était un type bien, mais je le respecte vraiment, il s'appelait Boris Mirononov. Quand on lui demandait, à l'époque où il était directeur du Rousskaïa kniga quels livres allaient plaire aux lecteurs, il répondait que la question était incorrecte. Pour lui, il ne s'agissait pas de faire des livres qui plaisent aux lecteurs, mais de former le bon goût des lecteurs. Il ne voulait pas imposer des goûts, mais il s'agissait de faire paraître des livres "de culture" et de bon goût. [...]

Face à la nouvelle situation, on a essayé de réagir. La première solution, ça a été d'augmenter le tirage des romans policiers. On a lancé une collection de romans policiers étrangers. Sans succès. Les autres, les "orthodoxes" préféraient la mort de la maison à cette entreprise honteuse. On n'a pas réussi à travailler seulement dans la logique marchande. Même si on a fait certaines choses. Comme dit notre directeur, ce qu'on a fait de plus bas, c'est la collection "Piorva" qui a connu de nombreuses rééditions et battu tous les records. Et aussi le Tsigane de Kalinine. Il y a peutêtre quelque chose d'intéressant dans tout ça, mais pas pour moi. Les gens achetaient bien, c'était un super-bestseller. Les rédacteurs qui travaillaient sur les bons livres étaient choqués, car on arrêtait ces parutions sous prétexte que leurs livres coûtaient cher. On leur disait : "Pour régler les salaires, il faut éditer le Tsigane." La "Piorva" faisait des tirages énormes, chaque famille a acheté des livres de la série. " (Rédacteur en chef d'une ancienne maison d'édition d'État, entretien réalisé en 1996.)

Le volontarisme désespéré engagé dans la publication de best-sellers par des professionnels ignorant et méprisant ce genre de production a pu conduire à des échecs. D'autant plus que, faute d'une véritable expertise et d'une capacité à anticiper, et plus encore à former et à fidéliser la demande sur le sous-secteur de la littérature populaire, les éditeurs tendaient à se précipiter tous sur le créneau (roman historique, policier, roman d'amour pour femmes) qui venait d'apparaître comme rentable. L'offre devenant très vite extrêmement importante, le créneau était déclaré saturé en deux ou trois ans : la vente pouvait rester élevée, mais elle était répartie entre de nombreux concurrents et le délai d'écoulement du stock était plus long. La littérature populaire, du point de vue des éditeurs, apparaissait comme une série de gisements vite épuisés, l'essentiel étant par conséquent de devancer les rivaux. Quant aux arbitrages entre gestion du capital symbolique et gestion du capital économique, ils se révélaient particulièrement ardus et, pour certains, très vite impossibles.

"Les maisons d'édition ont cru qu'elles pouvaient éditer de la littérature de masse, commerciale, en pensant que la littérature intellectuelle leur succéderait. Finalement, elles se trouvaient confrontées à un choix : soit continuer d'éditer des livres "commerciaux" et faire de l'argent, soit investir le bénéfice dans des projets éditoriaux moins rentables, où la circulation d'argent n'est pas aussi rapide mais où elles pouvaient garder leur renommée. La maison d'édition NordOuest, par exemple, s'est divisée en deux maisons, l'une pour les livres intellectuels, l'autre pour les livres de masse. La seconde contrôlait le système de diffusion des livres et imposait ses propres conditions aux intellectuels sur les tirages. Au bout d'un moment, la maison intellectuelle a dû se débrouiller seule. Dépourvue des moyens techniques, elle n'a pas réussi à survivre. " (Directeur d'une maison privée spécialisée dans les publications à haute valeur culturelle, entretien réalisé en 1996.)

Ce n'est pas un hasard si l'un des genres de publication qui s'est le plus développé en Russie concerne les encyclopédies en tout genre. La demande étant élevée, la publication d'encyclopédies permet à l'éditeur de concilier aisément vente de masse et idéal pédagogique, de retrouver dans le contexte actuel un idéal d'Aufklärung.

La plupart des éditeurs évoquent leur volonté de développer le marketing, terme qui subsume toutes les tentatives pour connaître les consommateurs et entrer 
en contact avec eux. Il peut s'agir d'organisations de rencontres avec les lecteurs, d'émissions de radio ou de télévision où le rédacteur en chef présente ses productions récentes. Les éditeurs à capital culturel élevé sont plus à l'aise dans ces démarches, qui poursuivent leur ancienne fonction d'éducateurs du peuple, que dans les discussions avec les "vendeurs ", qui indiquent sans ménagement ce qui est susceptible de se vendre et à quelles conditions.

\section{LA COURSE AUX SUBVENTIONS}

L'investissement de capitaux étrangers est quasiment nul dans l'édition russe. En revanche, dès la fin du système communiste, de multiples organismes étrangers (ministères de la Culture, fondations) ont entrepris de soutenir par des subventions l'édition et la diffusion de livres. Du point de vue des organismes fournisseurs d'aide, l'octroi de subventions permet à la fois de bien connaître le secteur, d'influer sur sa recomposition et de mener des politiques culturelles et idéologiques. Les ouvrages publiés avec l'aide de fondations étrangères ou de ministères - programme Pouchkine financé par la France, par exemple -, qui sont destinés au public intellectuel large ou restreint, visent à étendre le "rayonnement " d'une culture nationale ou d'une idéologie. Ce sont essentiellement des ouvrages occidentaux qui font l'objet de ces subventions. Les jurys de sélection des dossiers examinent d'ailleurs attentivement les curriculum vitae professionnels des traducteurs, qui doivent être bons connaisseurs de la langue originale et de l'œuvre. Les commissions de subvention permettent aussi de suivre assez finement les évolutions en cours et de connaître le fonctionnement des maisons. Tout dossier de demande d'aide fournit des indications sur l'entreprise, y compris sur des éléments financiers qui restent opaques pour le gouvernement russe. La Fondation Soros (International Science Foundation), implantée dans tous les pays de l'ancienne Europe communiste, a particulièrement développé des programmes destinés à soutenir la publication d'ouvrages de sciences humaines ${ }^{8}$. Elle a établi une liste d'ouvrages pour l'ensemble des pays dans lesquels elle intervient; les commissions locales qu'elle a mises en place peuvent en suggérer des modifications. Ces commissions locales sont composées d'universitaires qui avaient acquis antérieurement une certaine réputation comme chercheurs et traducteurs, ayant eu des relations avec le monde universitaire occidental et "parta- geant les valeurs de la Fondation ". La Fondation a également fait fonctionner, en 1995-1997, un programme d'aides à la publication de manuels d'enseignement supérieur. Il s'agissait de créer un nouveau standard de manuels, rompant radicalement avec les manuels soviétiques dans la forme et le fond, et directement repris du modèle occidental. Les éditeurs pouvaient concourir en présentant le texte d'un futur manuel dont l'impression était prise en charge par la Fondation. Le plus récent programme Soros annoncé en Russie concerne l'approvisionnement en livres des bibliothèques dont la situation est désastreuse, leurs capacités d'achat des nouvelles parutions étant très faibles. Le principe consiste en l'achat par la Fondation de tout le tirage d'un ouvrage vendu aux bibliothèque à $30 \%$ du prix la première année, $50 \%$ la deuxième et $75 \%$ la troisième.

En revanche, les aides attribuées à la production nationale par le gouvernement russe sont relativement modestes et portent surtout sur l'édition de manuels d'enseignement primaire et secondaire ainsi que sur quelques ouvrages scientifiques. Les modalités des aides à l'édition accroissent la marginalisation de la production autochtone de sciences humaines et sociales, car les éditeurs sont amenés à privilégier les traductions d'auteurs étrangers, qui non seulement se vendent mieux, mais sont à peu près seules susceptibles d'obtenir une subvention.

L'obtention d'une subvention auprès d'un organisme étranger met en jeu le capital social et culturel de l'éditeur. Il est en effet nécessaire de maîtriser la langue en usage dans l'organisme (anglais, français ou allemand), tant pour prendre les contacts nécessaires que pour constituer le dossier. Par ailleurs, il importe d'avoir des relations avec les décideurs, ou de savoir en nouer. Le minimum étant de savoir quel type d'ouvrage est susceptible de plaire à l'organisme subventionneur, plus exactement aux organismes, car il est usuel pour une maison de monter plusieurs dossiers simultanément. D'une manière générale, l'obtention d'une première subvention est un bon atout pour obtenir une nouvelle

8 - George Soros, d'origine hongroise, est parti s'installer en Angleterre en 1947, puis aux États-Unis en 1956. Ayant acquis une fortune considérable par la spéculation, il se présente comme un disciple de Karl Popper et un adepte de sa théorie de la "société ouverte . Dans son ouvrage, Le Défi de l'argent (Paris, Plon, 1996, pour la traduction française, première publication, anglaise en 1995), Soros déclare, à propos de son International Science Foundation: "Je suis inquiet. J'ai débloqué des fonds très importants - à elle seule, la Fondation m'a coûté plus de cent millions de dollars -, et si la Russie s'effondre, ce sera de l'argent en grande partie perdu... (p. 114). 
subvention - d'un autre organisme pour le même ouvrage, du même organisme pour un autre ouvrage. Ainsi s'est opérée rapidement une partition entre les éditeurs qui sont dans les réseaux de subvention et les autres. La publication d'un ouvrage subventionné est rarement susceptible d'accroitre directement le capital économique de l'entreprise. L'aide à la traduction ou à la publication concerne surtout des ouvrages peu rentables, parce que la vente est limitée ou bien s'effectue sur une durée trop longue pour les conditions actuelles de rentabilité. Les aides financières permettent surtout à l'éditeur de publier avec un faible débours et de ne pas être tributaire des retours rapides de vente. En revanche, l'obtention de subventions assure un accroissement du capital symbolique puisqu'elle vaut label de qualité dans une situation où les positions des éditeurs les uns par rapport aux autres sont encore à définir. En outre, la subvention peut valoir à l'éditeur un statut plus intéressant dans son emploi annexe ou principal (un universitaire éditeur ayant obtenu une aide d'une fondation étrangère peut le faire valoir pour sa carrière académique). Le traducteur d'un ouvrage subventionné voit sa réputation accrue, ce qui lui vaut de nouvelles commandes.

\section{L'ARGENT ET LA CULTURE}

Après la période d'exploitation intensive des "déficits " de l'ère soviétique et de la publication " tous azimuts ", les maisons survivantes ont commencé à se spécialiser, du moins à avoir une production dominante. Le secteur s'est polarisé avec à une extrémité des maisons à faible effectif (moins d'une dizaine de personnes), qui font de la "production pour producteurs " : ouvrages philosophiques, philologiques, historiques ou littérature d'avant-garde destinés à un public à très fort capital culturel. Les éditeurs sont des universitaires, des écrivains ou des artistes qui publient les ouvrages qui leur plaisent, à destination de lecteurs qui leur sont homologues. La maison d'édition et éventuellement la librairie associée font office de lieux de rencontre entre pairs. L'image de marque de chaque maison est étroitement liée à la reconnaissance des auteurs publiés en nombre limité. Ce pôle de la production restreinte est très dépendant de l'octroi de subventions et de la fidélisation de son public.

Les éditions Ad Marginem, créées en 1993, ont pour directeur un docteur en philosophie, ancien chercheur à l'institut de philosophie de l'Académie des sciences. Il dit avoir été en quelque sorte délégué par le groupe de chercheurs auquel il appartenait pour créer une maison privée puisque les structures étatiques se révélaient inadéquates pour la réalisation de nouveaux projets éditoriaux. La maison est spécialisée en philosophie et littérature "postmodernes ". Elle possède une double librairie (ouvrages en anglais et en russe) où sont organisées des rencontres intellectuelles. Elle compte, en sus du personnel de la librairie, trois employés.

À l'autre extrémité se situent des maisons faisant dans la " production de masse ", c'est-à-dire essentiellement dans le roman populaire, et comptant plusieurs dizaines d'employés. Leurs dirigeants sont eux aussi pour l'essentiel des diplômés de l'enseignement supérieur, mais issus de secteurs moins prestigieux que les précédents. Il peut s'agir aussi d'individus jeunes, certains étant encore étudiants ou lycéens au moment de la disparition du système soviétique.

La maison Olma-Press, créée en 1989, a pour directeur un ancien étudiant en philosophie. Avec quelques amis connus pendant ses années d'études, il a décidé de se lancer dans l'édition dès qu'il a été possible de démarrer des maisons privées. Après une première expérience sans grand succès (la publication d'un ouvrage de Freud), ils ont décidé d'éditer des romans populaires (livres d'aventure, policiers, thrillers). Ils ont engagé comme employés d'anciens professionnels de maisons d'État. Avec leurs bénéfices, ils ont pu monter leur propre système de diffusion opérant sur la Russie, sur d'ex-républiques soviétiques et même sur d'autres pays slaves. En 1996, ils déclaraient avoir publié 231 titres pour un tirage cumulé dépassant 6 millions de volumes. La maison compte 350 employés, dont 100 pour le réseau de diffusion. Le personnel affecté spécifiquement à l'édition est composé à $90 \%$ d'anciens rédacteurs de maisons d'État.

La position médiane est tenue par des maisons spécialisées dans une production "pratique " (manuels scolaires, par exemple) ou des maisons généralistes, dont les publications sont plus éclectiques et qui ont pour dominante des ouvrages dits sérieux, mais de bonne vente : encyclopédies, dictionnaires ou classiques de la littérature russe. Ces maisons comptent quelques dizaines d'employés, les éditeurs étant généralement d'anciens professionnels (rédacteurs ou cadres techniques des maisons d'État) ou bien d'anciens chercheurs en sciences exactes ou ingénieurs.

La maison Ladomir a été créée en 1990 par deux chercheurs de l'institut d'électronique d'une ville satellite de Moscou. Ils s'étaient connus durant leurs années d'études à cet institut. En 1990, ils ont décidé de changer de métier (leur secteur d'activité, lié à la recherche militaire, étant affecté par les changements politiques). Ils sont passés à l'édition, parce que, disent-ils, "ils avaient toujours aimé lire et avaient envie de s'occuper de ce qui leur plaisait ". L'affaire a été lancée par la publication des cuvres complètes de 
Louis Boussenard (écrivain français de la Belle Époque. spécialiste du roman populaire d'aventure pour la jeunesse et très apprécié du public en URSS). Les premiers fonds ont été réunis par souscription. Ont suivi les ceuvres complètes de Jules Verne et de Fenimore Cooper. Dès 1994, la maison a commencé la publication douvrages intellectuels. ce qui était le véritable projet des deux dirigeants, selon leurs déclarations, la publication de romans populaires n'ayant été que le détour nécessaire à la constitution d'un capital. Les premières publications de ce type ont été des rééditions d'ouvrages tombés dans le domaine public et relevant du secteur religieux (des textes sacrés de l'hindouisme). Puis Ladomir a proposé un partenariat de coédition à Naouka ("Science"), ancienne et prestigieuse maison d'État, dont la situation devenait critique. Le projet portait sur la très réputée collection du "Patrimoine littéraire ". Le rôle de Naouka n’a cessé de se réduire dans ce partenariat. La direction de Iadomir se flatte d'accorder un très grand soin à la qualité des textes publiés et au travail d'édition, qui garde les méthodes traditionnelles. Tous les manuscrits sont soumis à une correction stylistique et grammaticale poussée. Ladomir a même gardé la "correction à l'oreille ", aujourd'hui rarissime, qui met en jeu deux rédacteurs, l'un lisant le texte à haute voix tandis que lautre écoute et corrige. L'appareil critique des ouvrages publiés par Ladomir reprend les principes académiques mis en pratique dans les anciennes maisons d'État Naouka et Khoudojestvennaya literatoura (" Belles-Lettres "). La plupart des rédacteurs engagés par Ladomir viennent de ces maisons. Les dirigeants de Ladomir se targuent de viser systématiquement la plus haute qualité scientifique pour le choix et la réalisation de leurs ouvrages. Ils disent faire travailler des spécialistes universitaires de haut niveau ainsi que des traducteurs de qualité "à l'ancienne " et se pensent en "sauveurs " de la science russe. La maison publie 30 à 40 livres par an.

Avec cette amorce d'organisation, la redéfinition de la fonction éditoriale est devenue l'enjeu d'un conflit exprimé avec virulence dans la presse et qui se traduit par la mise en opposition entre "bon" et "mauvais" livre. Les anciens détenteurs d'un capital culturel élevé tentent d'en maintenir la valeur en stigmatisant fortement les livres "commerciaux " par le recours à des critères non seulement esthétiques, mais aussi éthiques, voire civiques. Ils continuent donc plus ou moins clairement à définir la fonction éditoriale par l'accomplissement d'une mission sociale, relevant d'un véritable apostolat :

"Avec nos convictions, nous ne pouvons pas nous per-
mettre des livres "commerciaux" comme le font certains;
nous ne pouvons pas publier des romans d'amour. Nous
refusons de faire des livres pour la simple consommation. Il
y a des livres qui se vendent bien. mais ce n'est pas de la lit-
térature. Les traductions, le contenu sont horribles. C'est un
péché d'appeler ça de la littérature. Moi. je ne veux pas
assumer ce péché. Passe encore que ça n'éduque pas la population, mais ça lui abîme le goût. Ils estropient la langue russe, il n'y a pas la moindre idée. Je ne veux pas dire qu'il faut imposer les bonnes choses d'une manière forcée, pourtant la bonne littérature par elle-même fait réfléchir les gens. Non seulement les livres "commerciaux" ne font pas réfléchir, mais ils stérilisent les capacités mentales. C'est comme si les gens fuyaient dans un substitut de la vie. Moi, je ne veux pas produire des choses comme ça. " (Responsable d'une petite maison d'édition spécialisée dans les ouvrages littéraires de qualité et les livres pratiques; ancien rédacteur d'une maison d'État; entretien réalisé en 1998.)

Les éditeurs du pôle " commercial " prennent part à la controverse moins en contestant l'opposition entre "bons " et "mauvais " livres qu'en invoquant le pragmatisme nécessaire dans une économie de marché (il faut éditer de "mauvais " livres pour avoir les moyens den faire de "bons") et la liberté du consommateur. À l'anathème jeté sur la production sans valeur éducative par les tenants de la conception à l'ancienne du métier, ils opposent le principe d'une hiérarchisation fondatrice. Certaines maisons d'éditions situées au pôle de la production de masse essaient d'ailleurs d'acquérir quelque crédit symbolique en lançant des collections d'ouvrages littéraires de qualité.

"Une maison d'édition, ça relève de l'économie de marché, elle doit faire du profit, se maintenir. Tout le reste, c'est de la foutaise, des illusions. Je pense à une maison d'édition... plus "commerciale" tu meurs. Eh bien! demain ils vont publier Anna Karénine. Et ceux qui exigeaient quion publie seulement des choses comme Anna Karénine et qui n'ont rien fait et seulement répété "C'est nous, la culture", aujourd hui ils ne peuvent rien publier. Et la maison en question, elle publiera encore autre chose après Anna Karénine. Bien sûr, ça dépend de ta culture intérieure si tu publies Anna Karénine ou si tu restes toujours sur le commercial. Moi, je ne connais pas une seule maison où les gens n'ont pas l'ambition de publier exclusivement de la littérature de bonne qualité et de contribuer à la culture russe. Mais avant il faut faire l'essentiel : construire les murs, le sol, le toit. Et c'est après seulement qu'on commence à faire le décor. " (Responsable d'une importante maison d'édition spécialisée dans le roman populaire, fondée par une équipe d'étudiants en philosophie, entretien réalisé en 1998.)

"La littérature de masse, la littérature de gare, ça doit exister. L'individu doit avoir la possibilité de satisfaire ses besoins. Je ne pense pas que celui qui lit de la littérature de gare soit pire que celui qui lit de la littérature hautement culturelle. C'est tout simplement différent. Et les gens ont besoin de choses différentes. Je ne parle pas de tout ce fumier qui prône la violence, le fascisme, de tout ce qui viole la volonté et la raison. Il me semble que ça, cest une maladie passagère qu il faut dépasser et pas interdire. L'individu doit avoir le choix de lire ceci ou cela et à la fin, les gens normaux, ils comprendront ce qui est bon. Ils ne vont 
pas courir après n'importe quelle ordure. L'essentiel, c'est que ce qui est bon soit gardé, que toute cette saleté ne repousse pas la bonne littérature de grande qualité. Tout doit être à sa place. Au bout du compte, nous avons besoin de fleurs et aussi de papier-toilette." (Responsable d'une maison d'édition qui a repris le titre de la maison fondée par ses ancêtres avant la révolution d'Octobre, entretien réalisé en 1998.)

Le conflit sur la définition de la profession, au demeurant, ne porte pas seulement sur la qualité des textes publiés, mais aussi sur la nature du travail effectué par l'éditeur. D'anciens rédacteurs de maisons d'État, qui refusent les nouvelles conditions d'exercice du métier, des universitaires ou des traducteurs réputés dénoncent le caractère selon eux bâclé et indigent du travail effectué par les maisons d'édition "commerciales". Et ils tendent à poser comme norme le processus de publication qu'avaient rendu possible, à l'époque soviétique, l'absence de concurrence entre maisons, l'indifférence aux coûts de production et le nombre élevé d'employés spécialisés.

"Quand on est un vrai rédacteur, on ne peut pas travailler dans les nouvelles maisons. Quand ces gens-là parlent de qualité, ils pensent à la beauté de la couverture. En pratique, le rédacteur y fait le travail du correcteur. Quand on commence à expliquer que chaque procédure doit avoir ses spécialistes, que le rédacteur ne peut pas lire le texte comme le correcteur, qu'on ne peut pas les confondre, on se heurte à l'incompréhension. Un vrai rédacteur ne publiera pas une ligne sans avoir vérifié les noms, les faits. Quand on dit aux éditeurs modernes qu'il faut faire ça, ils disent : "À quoi bon? L'auteur a déjà tout fait." Ça, c'est du bousillage. On ne peut éditer que des polars comme ça.
Leur principe, c'est que le livre doit être vendu bon marché et donc que le processus de production ne doit pas coûter cher. " (Rédactrice en chef dans une ancienne maison d'État, entretien réalisé en 1997.)

L'opposition entre l'argent et la culture fait assez généralement office de principe structurant dans la construction et le fonctionnement d'un champ autonomisé. Cependant, elle prend dans l'édition russe postcommuniste un tour particulier. D'une part parce qu'elle recouvre en bonne partie un clivage générationnel. D'autre part parce que persiste parmi les "anciens " professionnels une conception de la fonction éditoriale constituée à la période précédente - l'éditeur comme éducateur de masse -, qui contraste avec la prise en compte par les "nouveaux" des jeux et enjeux de la distinction culturelle. Enfin parce que la grande instabilité économique du pays fragilise particulièrement le secteur : l'édition a été sévèrement touchée par la crise qui a frappé la Russie à l'été 1998. Les plus durement atteintes ont été les maisons ayant une activité éditoriale importante et donc des productions en cours. Les vendeurs estimant que le prix des livres ne pouvait être sensiblement augmenté, et les profits escomptés étant laminés par la perte de valeur du rouble, ces maisons ont été amenées à suspendre leurs programmes de publication. Les petites structures ont été moins fragilisées. En tout état de cause, la hiérarchisation et la structuration, même partielles, de l'édition et de la librairie ne concernent que les deux plus grandes villes, la différence d'évolution entre celles-ci et le reste de la Russie n'ayant cessé de s'accentuer. 